\title{
OBSERVATIONAL TEST OF THE HOMOGENEOUS ARRAY CONCEPT: AN X-BAND IMAGE OF THE CRAB NEBULA
}

\author{
JUAN M. USON and T. J. CORNWELL \\ National Radio Astronomy Observatory, P.O. Box. 0, Socorro, NM 87801
}

\begin{abstract}
We have performed an observational test of the "Homogeneous Array" concept utilized in the MMA design, whereby the array elements are used in total power mode to provide the shortest spacings. Using the VLA in interferometric mode and a VLBA element measuring total power, we have reconstructed a mosaic image of the Crab Nebula, which fits the data adequately and is of good visual appearance.
\end{abstract}

\section{INTRODUCTION}

The proposed NRAO Millimeter Array (MMA) consists of forty 8 meter diameter telescopes configurable into a number of different arrangement yielding spatial frequency information on baselines ranging from meters to a few kilometers (see Brown, these proceedings). One of the prime observing modes of the MMA will be to mosaic images of large fields using multiple pointings of the array (e.g. Cornwell 1988, 1989). One of the recurring problems in imaging large fields of view has been the measurement of the short spacings, defined as baselines close to or much less than the element diameter $D$. It has been customary to use a single large element of diameter greater than $D$ for this purpose. The proposed MMA will measure the short spacings using the array elements themselves, acting both in total power and interferometric modes. It is straightforward to show theoretically that in the absence of systematic errors this must work; however, there remain some questions as to its feasibility under real observing conditions. Holdaway (these proceedings) has performed a series of simulations to address the possible problems. Here we describe an observational test using the VLA and a single VLBA element operating in total power. For our purposes the principal attraction of using the VLBA element is that the pointing errors at $8.4 \mathrm{GHz}(\sim 15$ arcseconds) scale to those specified for MMA elements at $230 \mathrm{GHz}$. Besides, the stability of the VLBA electronics make a total-power measurement feasible. While we cannot perform a comparison with a ground truth image, we have obtained an image consistent with both sets of data and with good visual appearance. 


\section{OBSERVATIONAL DETAILS}

The Crab Nebula subtends about 7 arcminutes which is slightly larger than the primary beam of a $25 \mathrm{~m}$ diameter telescope at $8.4 \mathrm{GHz}$ (5.4 arcminute FWHM). At the Nyquist sampling interval of about 2.7 arcminutes, a minimum of ten pointings are required to span the object with a respectable guard band. This, combined with its intrinsic brightness, makes the Crab Nebula an ideal target for a mosaicing test.

VLA observations These were kindly provided to us by Glen Langston. The Crab nebula was observed at $8.4 \mathrm{GHz}$ in the VLA D-configuration using 10 pointings at half-power spacings. These 10 pointings were observed in sequence 4 times, giving a total observing time of 18 minutes per pointing, and about 3 hours in total. The flux scale was established by referring the phase calibrator $0528+134$ to $3 \mathrm{C} 286$. Before imaging, the visibility data sets were phase rotated to a common phase reference position and the $u, v, w$ coordinates adjusted correspondingly.

VLBA observations We used the Los Alamos VLBA element for these tests. The VLBA elements are not instrumented for total power observations but we were able to obtain good data from the monitor information. We fixed the electronic gain and monitored total power (TP) and switched power (SP) during the run. TP alone is strongly dependent upon elevation but TP/SP is not. TP changes by a factor of 2 on observing the Crab, and is linear to about $2 \%$ over this range. We observed a 9 by 9 grid of pointings in azimuthelevation coordinates, spaced by 2 arcminutes, spending $25 \mathrm{~s}$ on each sample. Every 3 samples we moved to a point 20 arcmin away in azimuth for an offsource sample which provided the adopted zero-level. The total observing time per 9 by 9 grid was therefore about 40 minutes. Calibration was performed on 3C84 (whose flux was in turn derived from those of several Baars calibrators by Craig Walker), and the primary beam was measured using an 18 by 181 arcminute grid, also on $3 \mathrm{C} 84$.

\section{IMAGING}

A "standard" mosaicing algorithm (Cornwell 1988, 1989) was used to reduce the data. A maximum entropy algorithm (e.g. Narayan and Nityananda, 1986) is invoked to find one image which fits all the data constraints to within the noise level. The data constraints were, first, the VLA visibility data for each pointing, and second, the set of TP/SP measurements for the 9 by 9 grid observed using the VLBA element. The latter data were used directly rather than via an image as is often the case. The mosaicing algorithm relies upon a model for the primary beams of the elements used. For the VLA elements we use a polynomial model developed by Napier and Rots (1982), while for the VLBA elements we find a satisfactory model to be the primary beam corresponding to a uniformly illuminated, blocked aperture. We produced the three images shown in the figures. 


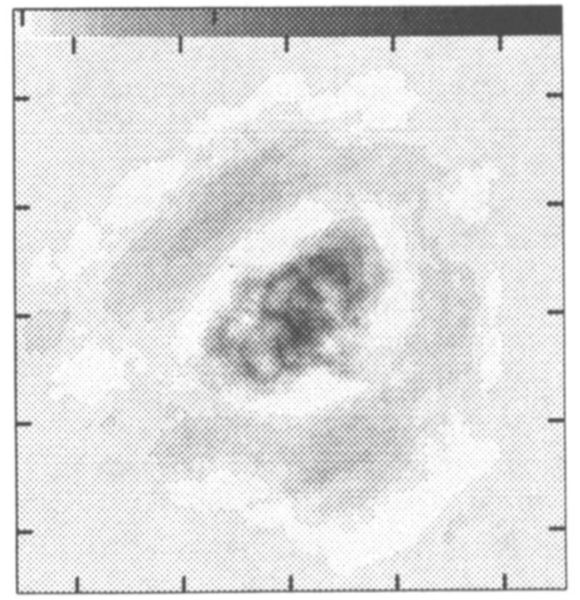

Fig. 1. Image made from only the VLA visibility data. The maximum brightness in the "bowl" is about $25 \%$ of the overall peak brightness. The reconstructed flux of the Crab Nebula is 253Jy. The display scale is linear over the range $-0.02,1.4 \mathrm{Jy} /$ beam and the tick marks are 200" apart.

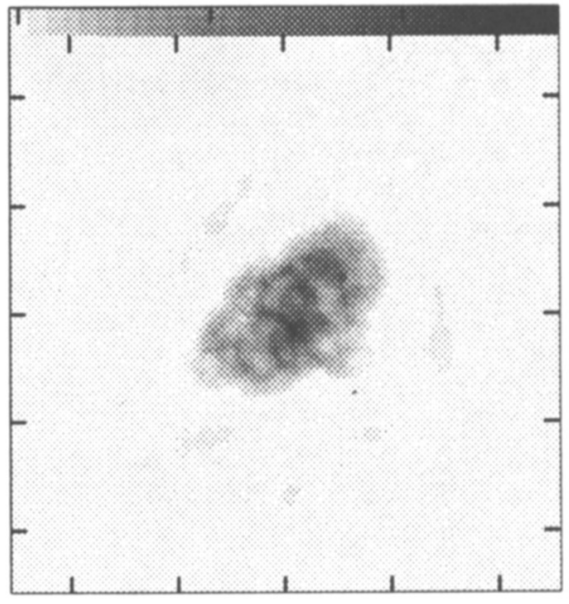

Fig. 2. Image made from only the VLA visibility data, using an edited version of Figure 1 (a) to define the support. The peak bowl brightness is about $4 \%$ of the overall peak brightness and the reconstructed flux is 331Jy. The display scale is as in Fig. 1. 


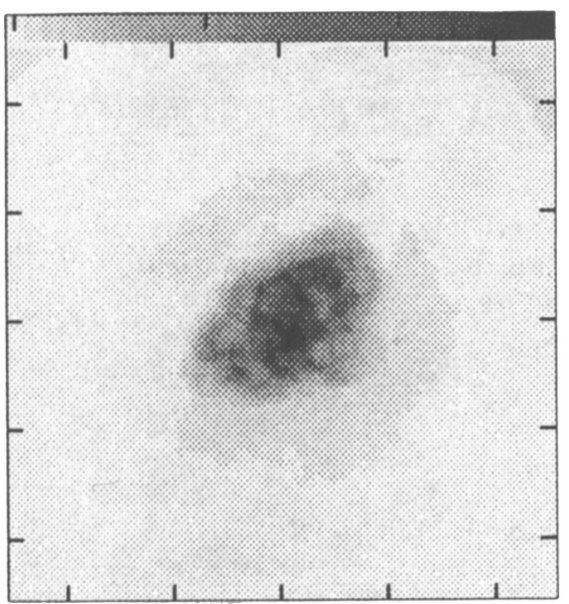

Fig. 3. Image made from both the VLA visibility and VLBA TP/SP data. The display scale is again as in Fig. 1. The peak bowl brightness is now only about $3 \%$ of the overall peak brightness: a level consistent with the systematic errors in the observation. The reconstructed flux is $498 \mathrm{Jy}$.

\section{COMMENTS}

- Despite the relative simplicity of our TP/SP observing scheme, we obtained a visually acceptable image which fits both data sets adequately.

- The level of residual noise off source ( $3 \%$ peak) is compatible with the level of non-linearity in the VLBA system (about $2 \%$ ) and the possible errors in cross calibration of the VLA and VLBA data (probably no better than 2-3\%). Errors in the pointing and in the assumed primary beam models will probably be important at lower levels than we reached in this experiment.

\section{ACKNOWLEDGEMENTS}

We are very pleased that we were able to use a VLBA antenna at the opposite end of the spatial frequency domain to its designed use. For this and their help and patience, we thank the VLBA project staff, especially Durgadas Bagri for his advice and Craig Walker for a timely measurement of the flux of $3 \mathrm{C} 84$. We also thank Glen Langston for providing the VLA data. This work is part of a larger program carried out in collaboration with Mark Holdaway; we thank him for numerous discussions and help with coding.

\section{REFERENCES}

Cornwell T.J., (1988), Astr. Ap., . 202, 316-321.

Cornwell, T.J. (1989), Proceedings of the Third NRAO-VLA workshop

on "Synthesis Imaging", Socorro, 1985, eds. Perley, R. A., Schwab, F. R., and Bridle, A. H. Pub. A.S.P.,

Napier, P.J. and Rots, A.H. (1982), NRAO test memo 134.

Narayan, R., and Nityananda, R. (1986), Ann. Rev. Astron. Astrophys. 24, 127-170. 